\title{
Experimental aortic valve heterotransplantation ${ }^{1}$
}

\author{
C. G. DURAN, A. J. GUNNING, AND R. WHITEHEAD \\ From the Nuffield Department of Surgery and the Gibson Laboratories, Radcliffe Infirmary, Oxford
}

\begin{abstract}
Pig aortic valves were transplanted into the descending aorta of 41 dogs. Ficin-digested valve grafts and fresh untreated grafts were associated with poor results and extremely short survival of the recipient animals. When freeze-dried valves were transplanted the mortality was reduced and the survival time increased, but there was a marked tendency for the grafts to rupture through the heterologous aortic wall cuff. If a homologous aortic cuff was used the survival time was increased, but rupture through the aortic wall cuff still occurred. The freeze-dried valve grafts eventually became almost acellular. Thrombus, which was commonly present on the valves, rarely showed organization. The valve cusps tended to remain thin and delicate, in one dog for as long as eight months after operation.
\end{abstract}

Homologous aortic valves have been transplanted into the dog (Beall, Morris, Cooley, and De Bakey, 1961 ; Duran, Manley, and Gunning, 1965 ; Lam, Aram, and Munnell, 1952; Murray, Roschlau, and Lougheed, 1956) and, more recently, into the human (Barratt-Boyes, 1964 ; Barratt-Boyes, Lowe, Cole, and Kelly, 1965 ; Binet, Duran, Carpentier, and Langlois, 1965 ; Davies, Lessof, Roberts, and Ross, 1965 ; Ross, 1963 ; and Ross, 1964). Aortic valve homograft transplantation in the human presents the difficulty of obtaining, shortly after death, a sufficient number of valves of varying sizes from young healthy donors. Heterologous aortic valves would solve this problem if they were shown to function successfully for a long time.

Carrel (1912) reported the four-year follow-up of a refrigerated segment of a human popliteal artery, transplanted into the abdominal aorta of a dog. Later, Hufnagel, Rabil, and Reed (1953) obtained good results in the dog and in the human with freeze-dried vascular heterografts. However, Creech, De Bakey, Self, and Halpert (1954), after using formalin-fixed and freeze-dried heterograft vessels in the dog and a freeze-dried hog aorta in a human, concluded 'that arterial heterografts preserved by the methods used in these experiments eventually weaken and dilate and therefore are unsatisfactory as vascular transplants'. Prosthetic materials have supplanted the use of vascular homo- or heterografts, but the results are far from satisfactory or, as Wesolowski (1962) puts it, 'acceptable at short term'.

Recently, Rosenberg, Henderson, Lord, Bothwell, and Gaughran (1962) have reported good

'Supported by a grant from the British Medical Research Council results with enzyme (ficin) treated vascular heterografts, and Marmor (1964 and 1965) is at present transplanting irradiated heterologous aortic valves into the dog. In this study we have been concerned with the fate of pig valves transplanted into dogs. The choice of the pig as the donor animal was made because of the size and availability of pig aortic valves.

\section{TECHNIQUE AND MATERIAL}

Because of the technical difficulties involved in an orthotopic transplant, the valves were placed in the descending aorta of the recipient animal as in previous experiments using homografts (Duran et al., 1965). After insertion of the graft aortic incompetence was produced in all the animals. Arterial pressure tracings were recorded above and below the graft, before and after producing aortic incompetence.

The pig valves were obtained unsterile, immediately after death, and were kept in physiological saline in a refrigerator at $4^{\circ} \mathrm{C}$. for up to 24 hours.

Two methods of preparing the grafts were employed. In the first, the aortic valve was dissected, leaving $1 \mathrm{~cm}$. of ascending aorta and as little as possible of the myocardial aspect of the valve. The coronary arteries were ligated at the origins. A separate cuff of heterologous donor ascending aorta was anastomosed to the myocardial aspect of the valve in order to facilitate its insertion into the descending aorta of the dog (Fig. 1). The valves so prepared were kept in Gross's solution (Gross, Hurwitt, Bill, and Peirce, 1948). These grafts were then divided into four groups and implanted (1) after irradiation with a 
single dose of 2,000 rads; (2) after ficin digestion, according to the method of Rosenberg $e t$ al. (1962) and Lord (1963); (3) fresh non-treated valves; (4) after sterilization by liquid ethylene oxide, freeze-drying, and storage under vacuum at room temperature.

In the second method the pig aortic valve was completely dissected so that only the three cusps remained together with $2 \mathrm{~mm}$. of adjacent aortic wall. The heterograft was then sutured in a subcoronary position in an aortic segment from another donor dog (Fig. 2). A cuff of ascending aorta from the same donor dog was anastomosed to the myocardial aspect in the usual way. These grafts, constituting group 5 , had therefore a heterologous valve within a homologous cuff, and were then sterilized, freeze-dried, and stored before implantation as in group 4 .

\section{RESULTS}

The irradiated valves were found to be so friable that it was impossible to implant them satisfactorily. The ficin-treated valves were strong but had lost some of their pliability. Three such valves were transplanted into dogs. One died on the operating table, and the remaining two died four and 20 hours after surgery. Both had massive thrombus formation in the region of the heterograft and this series was abandoned. Equally disappointing results were obtained using fresh untreated heterologous valves. In three cases, two survived for three and four days, and the other died after three hours with intracardiac thrombus due to endocardial damage caused during the production of aortic incompetence. In the longerterm survivors, marked thrombus formation in the valve sinuses was present. Because of our previous experience with fresh homologous valves (Duran and Whitehead, 1966) and because of the poor results obtained when fresh pig valves were transplanted into 13 calves (unpublished), this series was also abandoned.

Better results were obtained using freeze-dried grafts. In 24 dogs, the valve and the aortic cuff were heterologous. In this series there were seven operative deaths. One occurred during the induction of the anaesthetic, five were due to complications occurring during the production of host aortic incompetence, and one died of haemorrhage at the end of the operation. Nine dogs died after between six and 36 hours, four of haemorrhage, three as a result of the aortic incompetence, two in a decerebrate state, and one of an unknown cause. Eight dogs died suddenly $9,12,15,16,16$, 30,30 , and 50 days post-operatively. In six of

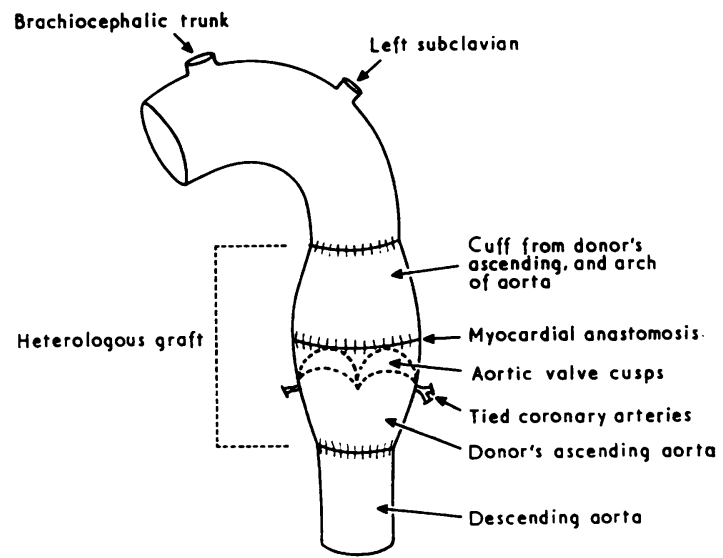

FIG. 1. Technique of insertion of heterologous aortic valve graft.
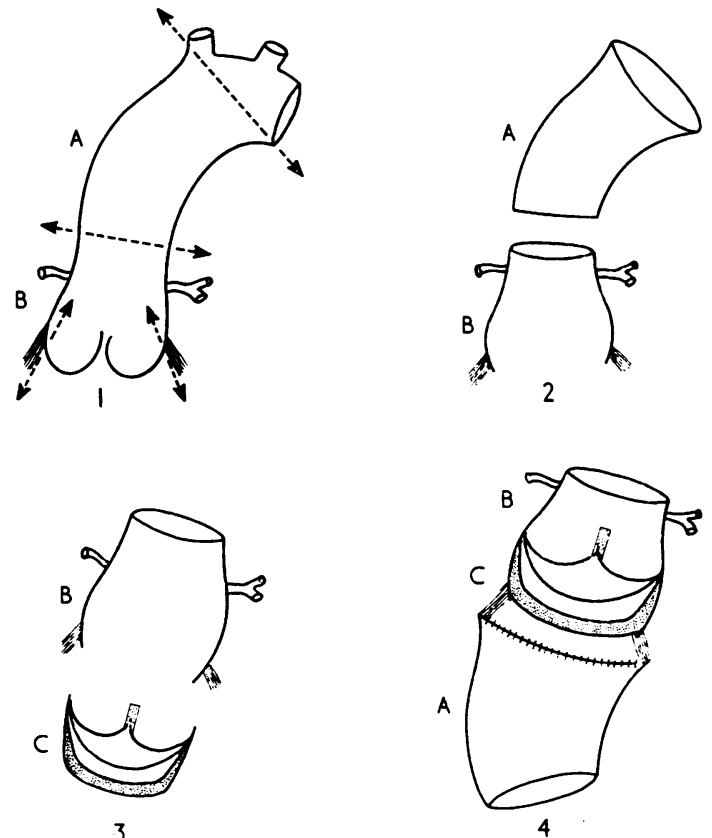

FIG. 2. (1) Donor dog aorta and valve. Lines indicate site of section. (2) Donor dog aortic wall cuff $(A)$ and aortic ring cuff (B). (3) Aortic ring cuff of donor dog $(B)$ and prepared pig aortic valve $(C)$. (4) Graft ready for implantation. Pig valve $(C)$ in situ with complete dog aortic wall cuff. Note that largest diameter of $A$ is anastomosed to myocardial aspect of $B$. 


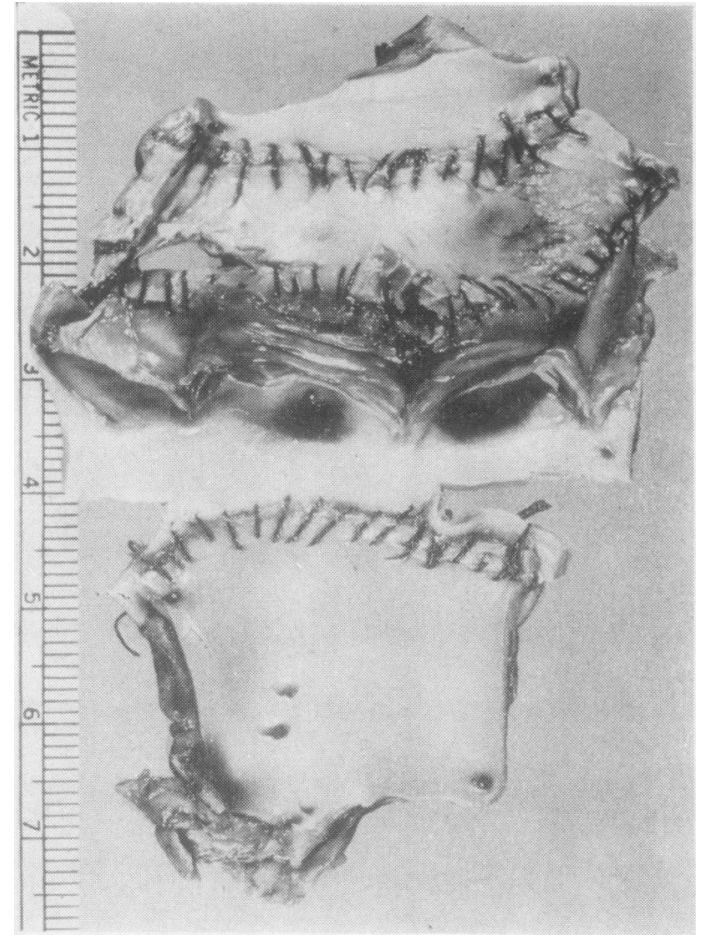

FIG. 3. Freeze-dried pig valve and pig aortic wall cutf 16 days after transplantation. Observe ruptures in aortic wall cuff and at its anastomosis with the valve.

these there was a rupture of the aortic wall cuff. The remaining two animals died at 15 and 16 days and had tears at the anastomosis between the myocardial aspect of the valve and the aortic cuff, in addition to rupture of the aortic cuff itself (Fig. 3). Macroscopically, the valve cusps were thin and pliable in all but three specimens. In these three, the valve cusps appeared slightly thickened but were still mobile, and approximation of the cusps was good. Thrombus, however, was present in the valve sinuses of all but two dogs.

Because of the deaths due to rupture of the heterologous aortic wall cuff, the pig valve was incorporated into a dog aortic cuff. Eleven dogs had such grafts; three died a few hours postoperatively, two of haemorrhage and another of suspected embolism from a thrombus on the torn host aortic valve. A further dog died of an unknown cause at two days, another of haemorrhage at three days, and another of traumatic mitral incompetence at seven days. Four dogs died of rupture of the homologous aortic wall, 3 , 6,18 , and 30 days post-operatively, and one dog was sacrificed at eight months because of the presence of an aneurysmal dilatation of the homologous aortic cuff. Macroscopically, all the valves $\frac{\overline{\bar{O}}}{\overline{0}}$ appeared to be in perfect condition except where $\frac{\text { D }}{\vec{D}}$ tearing had occurred in those with rupture of the $\unrhd$ homologous aortic wall. Macroscopic thrombus is in the aortic sinuses was seen in two dogs which $\overrightarrow{0}$ died on the third day.

HISTOLOGY Only the freeze-dried transplants $\stackrel{\omega}{\mathscr{S}}$ were examined histologically. The grafts were $\vec{x}$ fixed in formalin and representative blocks were i processed through paraffin. Sections were cut at $\stackrel{N}{\sigma}$ $5 \mu$ and stained with haematoxylin and eosin or (H. and E.).

The graft prior to transplantation The freeze- $\stackrel{-}{2}$ dried valve cusp contains the nuclei of fibrocytes $Z$ and has flattened endothelial cells on its surface. The aortic cuff exhibits perinuclear vacuolation $\frac{\mathbb{\Phi}}{3}$ and often has microcysts in the media which $\underset{\mathbb{D}}{\vec{D}}$ probably represent ice crystal spaces. The nuclei of the cellular component of the aorta and valve $\mathscr{\mathscr { Q }}$ show a pattern of shrinkage and slight pyknosis. comparable to that seen in early ischaemia. Similar nuclear changes are also present in the small tags of cardiac muscle which remain at the valve base. The valve base tissue adjoining the cardiac muscle is mainly fibrohyaline in nature, $\mathbb{D}$ but it also contains areas which have a more or $\underset{\vec{F}}{\vec{F}}$ less distinct cartilaginous appearance.

Heterologous grafts After only six to 12 hours increasing nuclear lysis of the cellular component of the valve was apparent, and between three and six days it was almost complete. Some nuclei $\underset{\sim}{\tilde{N}}$ persisted, however, near the tip or just under the surface, where, in addition to occasional swollen 3 . fibroblasts, a few polymorphs and histiocytes were seen. In one case at nine days marked polymorph 3 infiltration was seen, especially at the valve base, 0 but in this dog a Gram strain revealed large colonies of Gram-positive cocci throughout the valve tissue and adjacent aortic wall. In another $\operatorname{dog}$ at 12 days there was also a polymorph in- $\tilde{N}$ filtration in excess of usual. This was present just $N$ under the surface of the valve and in the free $N$ margin, but in this dog no organisms were seen. $\omega$ In two other dogs which died at 30 and 50 days most of the valve was cellular. The cells were a mixture of polymorphs, lymphocytes, histiocytes, and occasional plasma cells. Near the free margin fibroblast nuclei were also present, but in other $\frac{0}{0}$ areas these had disappeared. On nearly all the $\frac{\mathbb{D}}{0}$ valves recent thrombus was seen (Fig. 4), some- $\frac{?}{\mathbb{D}}$ times as thin surface flecks but often as larger, $\unrhd$ more obvious masses (Fig. 5). In only two dogs 
which died after 30 and 50 days was the thrombus showing signs of organization (Fig. 6). In both, this consisted of a fibro-endothelial reaction to valve surface thrombus together with organization of a larger mass occupying the valve sinus. In the dog which died at 50 days, the reaction was continuous over the surface of the thrombus in the sinus with a fibro-endothelial proliferation on the adjacent aorta. This soon faded out into the usual appearance of an acellular aorta without an endothelial lining.

The tissues at the valve base showed a picture of gradual nuclear loss. The cardiac muscle exhibited an eosinophilia and loss of cross striations, changes identical with cardiac infarction. At about five days a polymorph zone at the outer edge of the cardiac muscle was well established (Fig. 7). After a week histiocytes appeared and an early invasion by granulation tissue was beginning. A cellular fibrous replacement of the outer part of the cardiac muscle remnant was seen at 50 days.

Most of the nuclei of the intimal cells and medial muscle cells in the aortic graft also disappeared, only small numbers of muscle cell nuclei persisting in a subintimal site. On the inner surface, a thin film of recent non-organizing thrombus was commonly present. Elastic tissue in the aorta largely persisted with an unchanged pattern except in small foci mostly in the outer third of the media. Here it appeared to undergo dissolution associated with a slight inflammatory cell infiltration. In one dog which died at 50 days these areas were more than usually densely infiltrated and also contained histiocytes and occasional giant cells. In some areas this change was associated with a small plaque of overlying fibro-endothelial proliferation on the aortic intimal surface (Fig. 8). This plaque was continuous with similar tissue bridging the aorta to aorta anastomosis. The fibro-endothelial bridge was the only evidence of union in this group of cases.

After 24 hours the adventitia began to exhibit an inflammatory cell infiltration. At first this was mainly polymorphonuclear, but at one week lymphocytes, plasma cells, and histiocytes appeared and a little later fibroblast and capillary proliferation was apparent, so that the adventitia resembled young granulation tissue (Fig. 9). At about two weeks plasma cells, lymphocytes, and histiocytes were the predominant cell types, but in long-term survivors a decrease in numbers with a tendency to a focal distribution was noted. The density of the infiltrate varied from valve to valve and the valves examined at nine, 12 , and 30 days contained a foreign body giant-cell reaction. This consisted of multinucleate cells and a focal histiocyte reaction in relation to birefringent particulate material distinct from sutures. An occasional suture also showed a similar reaction, but the majority of sutures in these three dogs and those in all the remaining dogs did not evoke such a reaction.

Heterologous valve with homologous cuff With only occasional differences, the changes were similar to those seen in the total heterografts. The impression was that the cellular infiltrate in the adventitia was less dense than in the previous group. In the valve examined after 30 days, however, a dense infiltrate was seen but it was associated with a foreign body reaction similar to that seen in the total heterografts. The valve cusps in this group remained thin but became for the most part acellular except for a few fibroblasts, degenerate polymorphs, and an occasional histiocyte near their free margin or just under the surface. Thrombus of variable amount and extent was commonly present on both valve and aortic wall, but its organization was never seen (Fig. 10).

In the eight-month survivor a few fibroblast nuclei were all that persisted in otherwise thin, acellular valve cusps (Fig. 11). In this case a Teflon insert was placed behind the valve base and there was a mild histiocytic reaction to it with an occasional multinucleate giant cell. In the adventitia and in relation to the sutures a giantcell reaction was not seen and the mainly acellular aortic wall showed only patchy fibrous replacement of areas of elastic tissue. These fibrous areas were the site of ectasia. At the aorta to aorta anastomosis a simple fibrous union had occurred, the elastic fibres ending abruptly at the ends of the aorta.

Encouraged by these experimental results we decided to prepare some sow's aortic valves ready for use in patients who might require a particularly large valve.

On 10 October 1963 a patient suffering from syncope, dyspnoea, and angina because of aortic incompetence and stenosis was operated upon and a freeze-dried sow's valve was transplanted. The patient died 12 hours later from persistent bleeding. At necropsy the sow valve was found to be competent and secure in position.

Since this time four further cases have been treated. Two are still alive nine months and four months after operation. In the patients who died the valve did not contribute to the cause of death. 


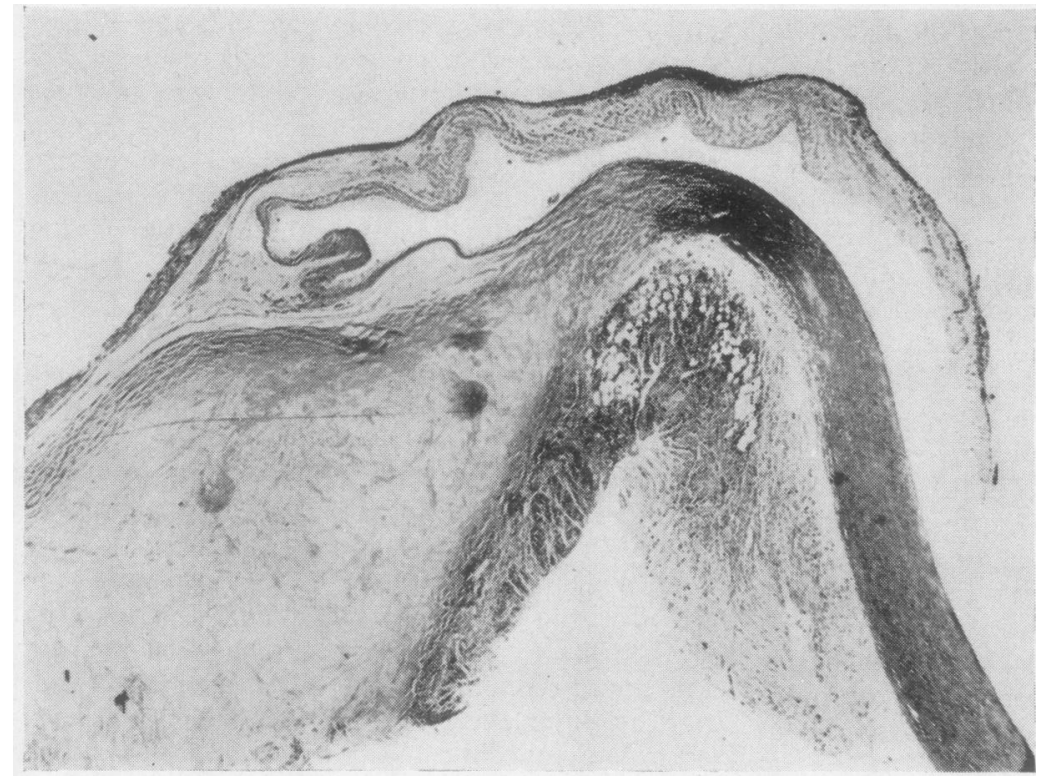

FIG. 4. Twelve days post-operatively. Note surface film of thrombus, relative acellularity of valve cusp, and fibrohyaline tissue of valve base. $H$. and $E$. $\times 20$.

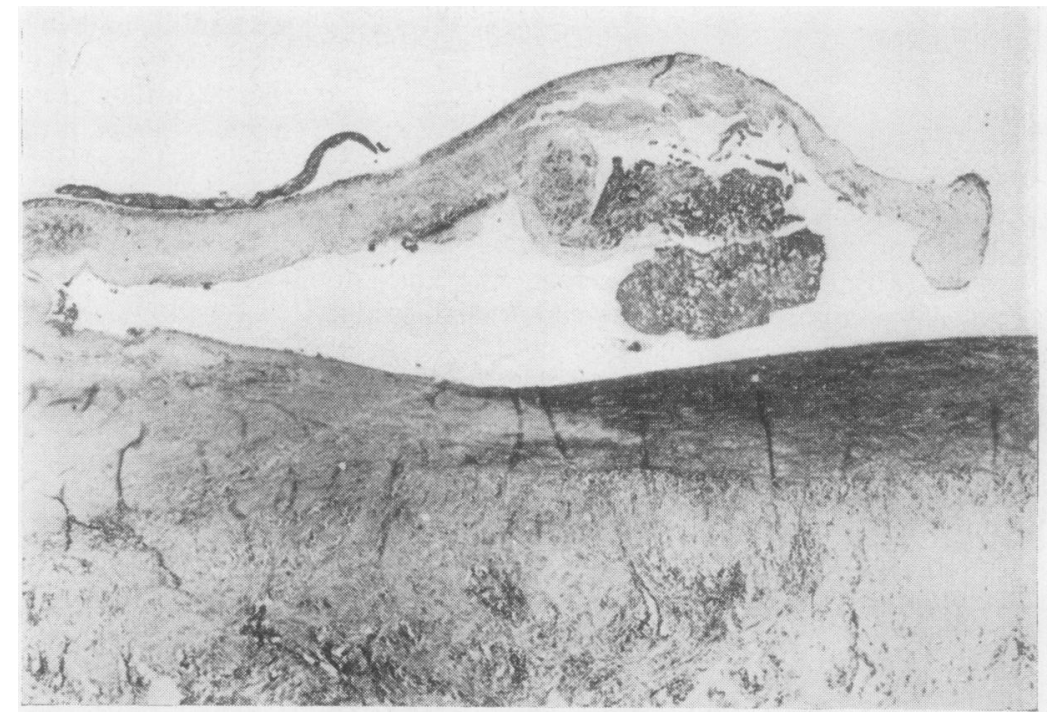

FIG. 5. Thirty days post-operatively. Surface film and larger mass of thrombus in valve sinus. $H$. and $E . \times 20$. 


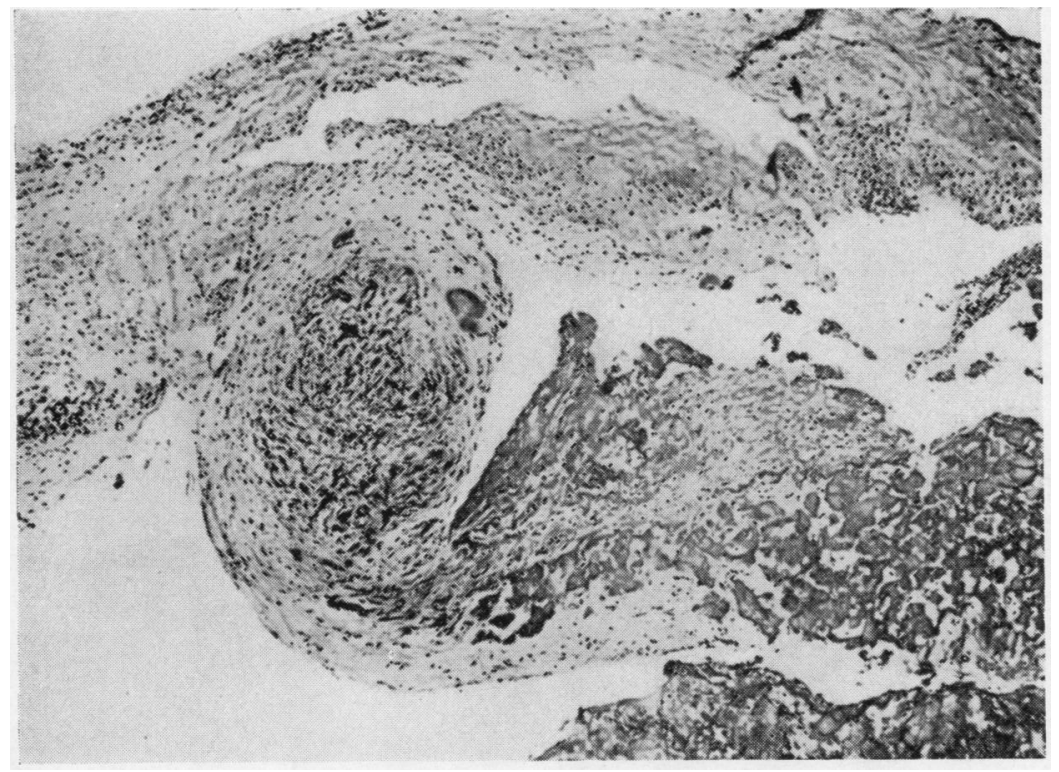

FIG. 6. Shows detail of Figure 5. Thrombus is organizing and valve cusp is cellular. $H$. and $E$. $\times 60$.

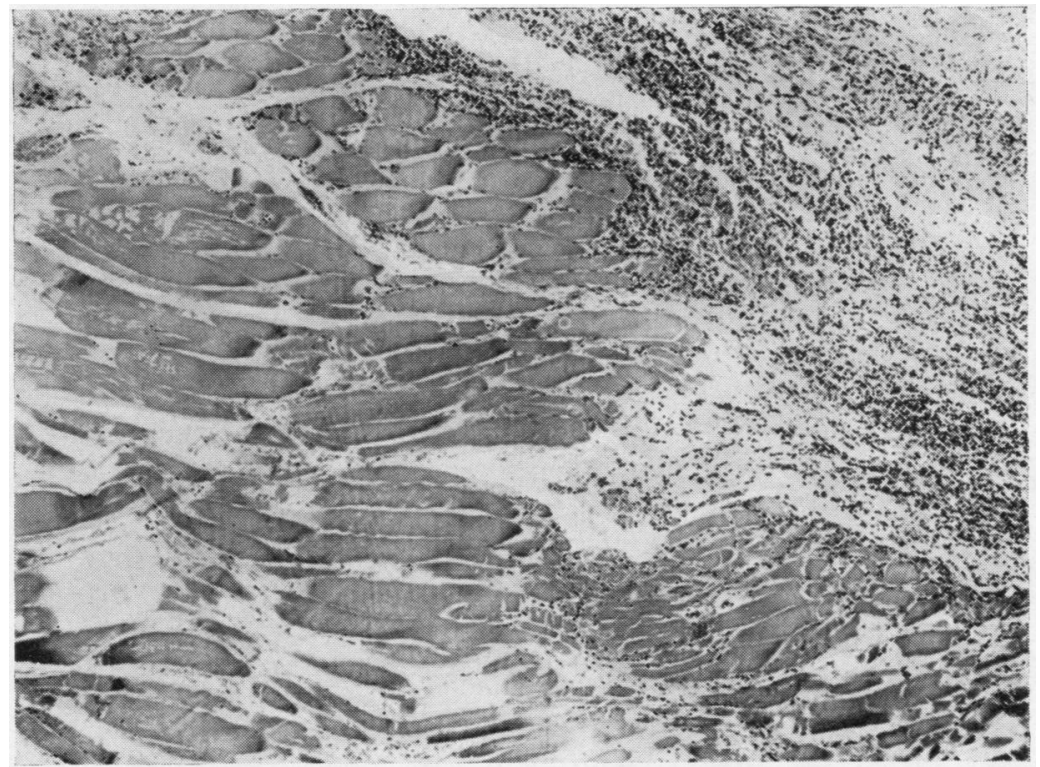

FIG. 7. Five days post-operatively. Outer edge of cardiac muscle in valve base. Note infarcted appearance and polymorph leucocyte zone. $H$. and $E$. $\times 60$. 


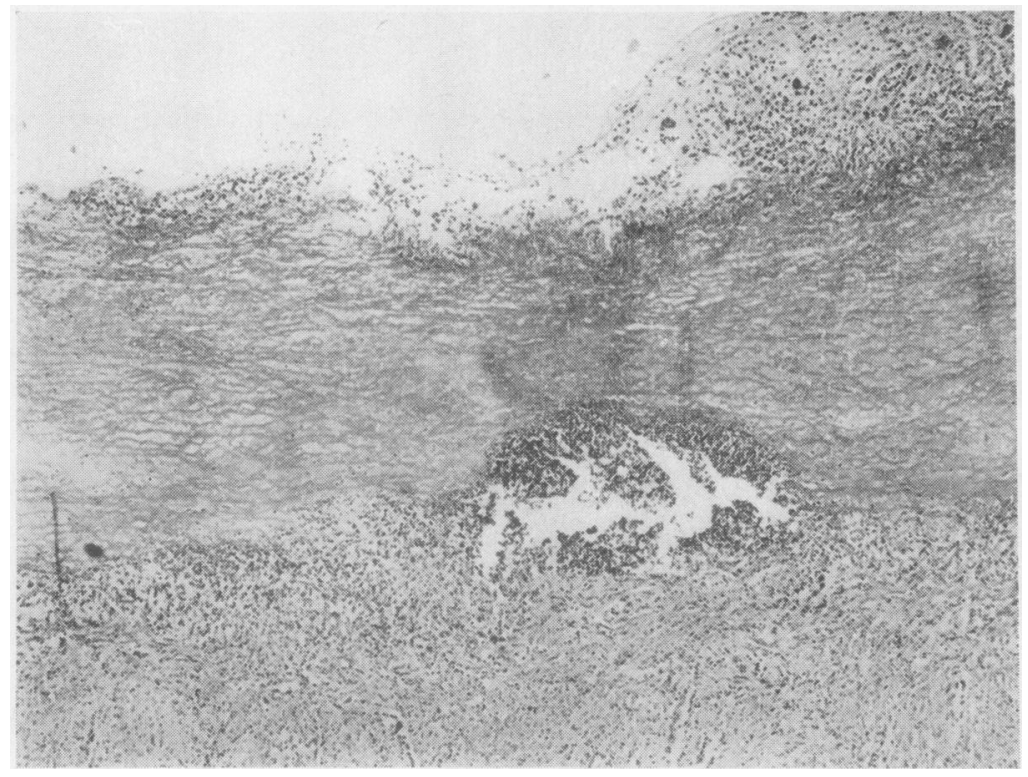

FIG. 8. Fifty days post-operatively. Inflammatory-cell foci in outer media continuous with adventitial reaction. Note intimal fibro-endothelial reaction with giant cells and acellular appearance of most of remainder of aortic wall. $H$. and $E . \times 45$.

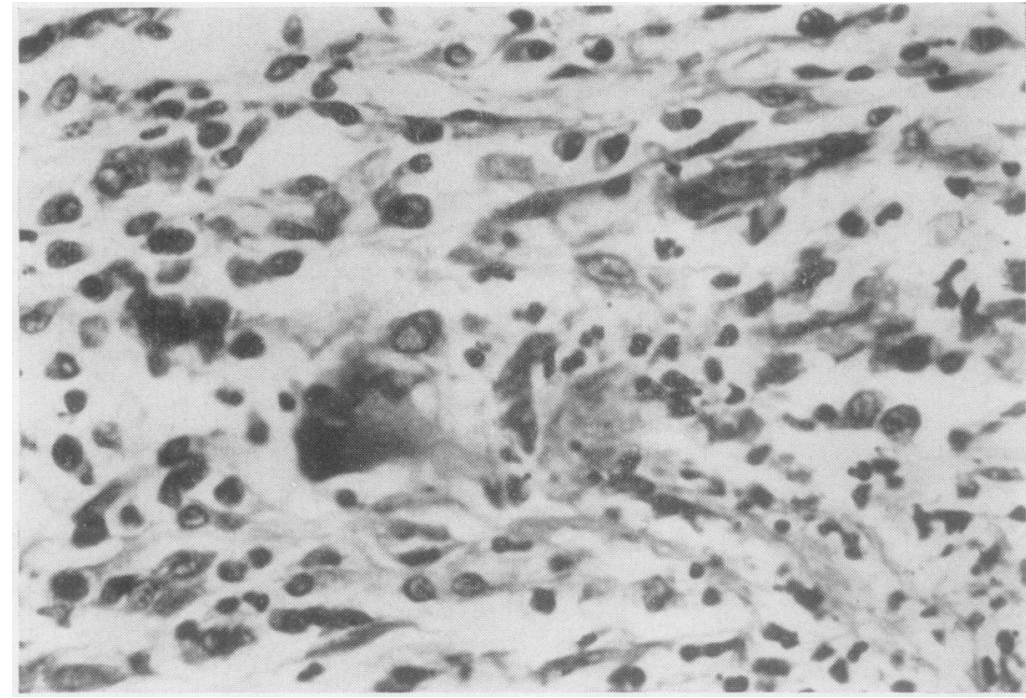

FIG. 9. Nine days post-operatively. Adventitial reaction showing a mixed inflammatory cell infiltrate, fibroblast proliferation, and poorly formed multinucleate giant cells. $H$. and $E . \times 500$. 


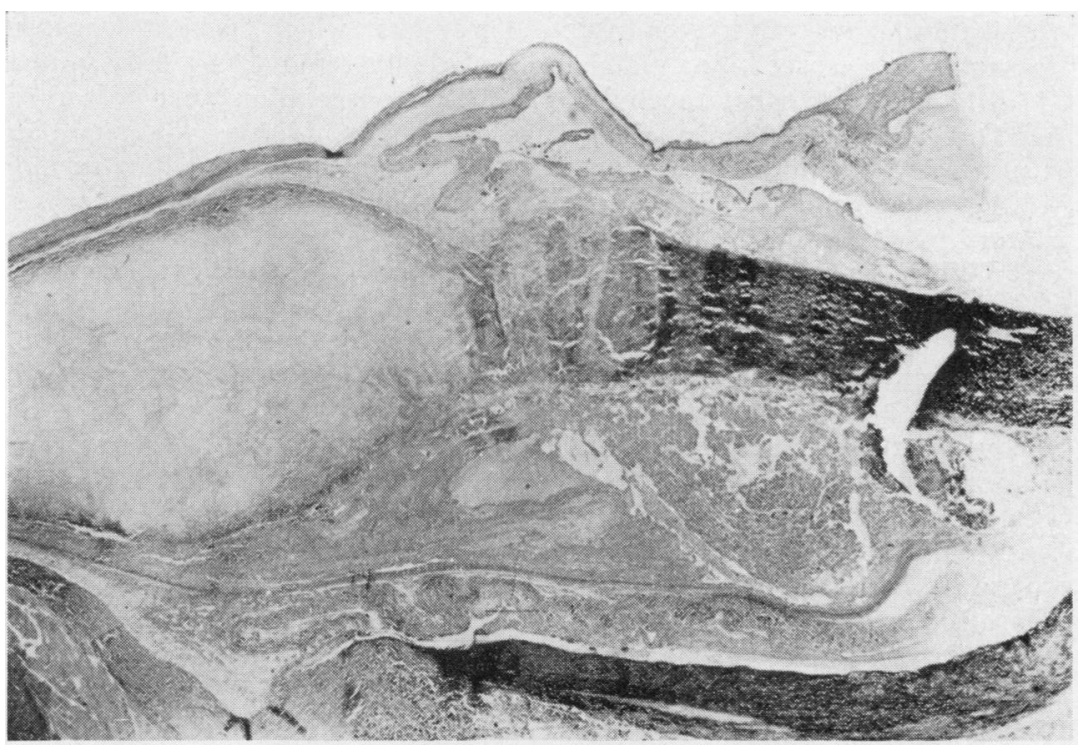

FIG. 10. Eighteen days post-operatively. Thin, acellular valve cusp with pseudocartilaginous appearance of tissue at valve base and non-organizing thrombus on valve cusp and aortic wall. $H$. and $E . \times 20$.

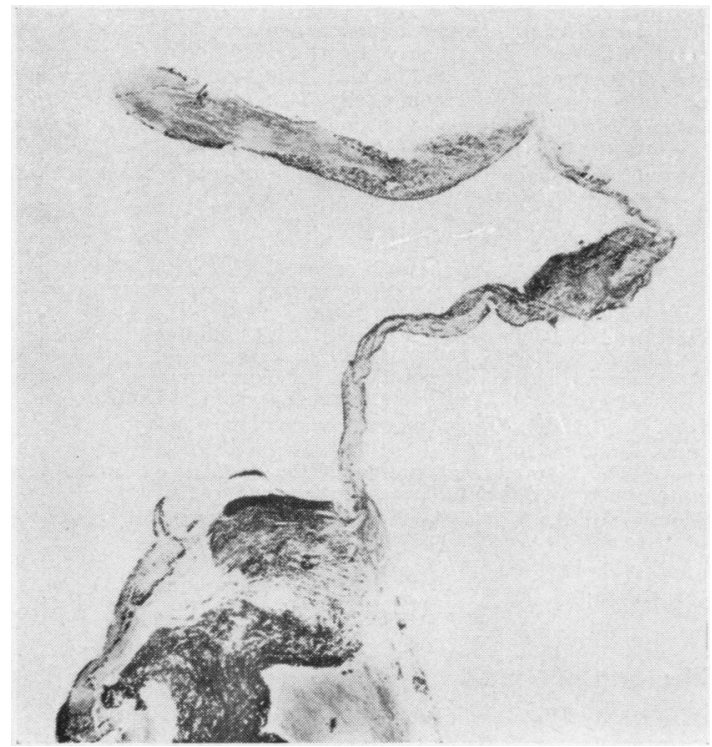

FIG. 11. Eight months post-operatively. Thin valve cusps, acellular except for a few foci of fibroblasts. $H$. and $E$. $\times 20$.
In September 1965 the first successful aortic heterograft transplantation was done by Professor Binet and one of us (C. D.) (Binet et al., 1965) using a sow valve prepared in Oxford. Since this time Professor Binet has done 27 more cases. The results of these will be the subject of another report.

\section{DISCUSSION}

In our hands, ficin-digested and irradiated valve grafts compare poorly with freeze-dried specimens. The poor results with the transplanted ficin valves and the friability of the irradiated valves, which precluded transplantation, may be due to our relative inexperience with the techniques involved. Freeze-drying appears to be the method of choice in the preparation of the grafts, and the results when the grafts are transplanted are even superior to those when fresh valve grafts are used. We have discussed elsewhere (Duran and Whitehead, 1966) the probable reason for this in a comparison of results using fresh and freeze-dried homologous valve grafts. 
One freeze-dried heterologous valve, even after eight months' transplantation, was thin, pliable, and functional, although somewhat acellular. Allowing for haemorrhage and other operative deaths, the short survival of the dogs that received freeze-dried, total heterologous grafts seemed to be related to rupture of the graft in the region of the aortic cuff. When it was replaced by a homologous cuff, death was still prone to occur in this way, although the tendency to rupture decreased. The dog which survived eight months had a reinforcing Teflon tube outside the graft in the region of the valve. This indicates that the weakness of the aortic wall may be overcome by this method. Histologically, the impression was of an increased adventitial inflammatory reaction in the total heterografts. It is possible that the reaction determines the degree of aortic wall involvement and that, when severe, this may predispose to rupture. Moreover, from experience with homografts (Duran et al., 1965 ; Duran and Whitehead, 1966), such changes appear to precede atherocalcific degenerative changes if long survival occurs. One is tempted to suppose that a homologous aortic wall would be less likely to excite a graft-immune response or that the degree of excitation would be less. On the other hand, an equally important factor in the damage to the aortic cuff may well be the inflammatory response to operative trauma, infection of the site, or contamination by foreign body. The latter appears to have occurred in several of the cases examined.

The majority of the valve cusps remained in good condition, gradually becoming acellular. This feature is in part probably related to the freeze-drying, since in both homologous and heterologous valve transplantation it occurs much more rapidly than after transplantation of fresh valves. In turn the loss of nuclei appears to account for its relative non-reactivity to complicating surface thrombus, the organization of which could obviously lead to valve distortion.

In these experiments organization of valve thrombus occurred in only two cases, when total heterografts were used. Thrombus formation in both the freeze-dried series is possibly explained by factors other than the heterologous nature of the graft. A poor mechanical result, operative trauma, and the inflammatory reaction consequent upon infection or wound contamination may be the underlying causes. Repeated thrombus deposition could ultimately stimulate its organization by viable cells remaining in the graft, by cells migrating from neighbouring host tissues, or even by deposition of cells from the circulating blood.
Thrombus which is not organized is in allo probability removed by lytic mechanisms.

These observations lead to the conclusion that, $\frac{\overline{5}}{\overrightarrow{5}}$ given ideal conditions, the heterologous valve can $\stackrel{\mathbb{}}{\AA}$ survive and function for long periods. Our failures were more related to rupture of the aortic cuff, $\vec{P}$ and with improved technique this could probably? be avoided. These disadvantages are, however, not $\vec{\omega}$ applicable if the heterologous valve is transplanted $\stackrel{\circ}{\circ}$ in the subcoronary position where the use of an $\vec{x}$ aortic cuff is not required.

\section{REFERENCES}

Barratt-Boyes, B. G. (1964). Homograft aortic valve replacement in aortic incompetence and stenosis. Thorax, 19, 131.

Lowe, J. B., Cole, D. S., and Kelly, D. T. (1965). Homograft valve replacement for aortic valve disease. Ibid., 20, 495.

Beall, A. C., Jr., Morris, G. C., Cooley, D. A., and De Bakey, M. E. Z (1961). Homotransplantation of the aortic valve. J. thorac. cardiovasc. Surg., 42, 497.

Binet, J. P., Duran, C. G., Carpentier, A., and Langlois, J. (1965). Heterologous aortic valve transplantation. Lancet, 2, 1275.

Carrel, A. (1912). Ultimate results of aortic transplantations. J. exp. $\frac{\mathbb{D}}{-}$ Med., 15, 389.

Creech, O., De Bakey, M. E., Self, M., and Halpert, B. (1954). The $\overrightarrow{0}$ fate of heterologous arterial grafts: an experimental study. Surgery, 36, 431.

Davies, H., Lessof, M. H., Roberts, C. I., and Ross, D. N. (1965). Homograft replacement of the aortic valve. Lancet, 1, 926.

Duran, C.G., Manley, G., and Gunning, A. J. (1965). The behaviour of homotransplanted aortic valves in the dog. Brit.J.Surg., 52, 549. and Whitehead, R. (1966). Experimental homologous aortic valve transplantation: a comparison between fresh and freeze-o dried grafts. Ibid., 53, 1049.

Gross, R. E., Hurwitt, E. S., Bill, A. H., and Peirce, E. C., 2nd (1948). Preliminary observations on the use of human arterial grafts in the treatment of certain cardiovascular defects. New윽 Engl. J. Med., 239, 578.

Hufnagel, C. A., Rabil, P. J., and Reed, L. (1953). A method for the preservation of arterial homo- and heterografts. Surg. Forum, 4, 162.

Lam, C. R., Aram, H. H., and Munnell, E. R. (1952). An experimental study of aortic valve homografts. Surg. Gynec. Obstet., 94, 129.

Lord, G. H. (1963). Johnson and Johnson Research Foundation, New Brunswick, New Jersey, U.S.A. Personal communication.

Marmor, L. (1964). The repair of peripheral nerves by irradiated homografts. Clin. Orthop., 34, 161.

(1965). University of California, Los Angeles, U.S.A. Personal communication.

Murray, G., Roschlau, W., and Lougheed, W. (1956). Homologous aortic-valve-segment transplants as surgical treatment for aortic and mitral insufficiency. Angiology, 7, 466.

Rosenberg, N., Henderson, J., Lord, G. H., Bothwell, J. W., and Gaughran, E. R. L. (1962). Use of enzyme-treated heterografts as segmental arterial substitutes; V. Influence of processing factors on strength and invasion by host. Arch. Surg., 85, 192.

Ross, D. N. (1963). Surgical reconstruction of the aortic valve. Lancet, 1, 571 .

(1964). Homotransplantation of the aortic valve in the subcoronary position. J. thorac. cardiovasc. Surg., 47, 713.

Wesolowski, S. A. (1962). Evaluation of Tissue and Prosthetic N Vascular Grafts. Thomas, Springfield, Illinois.

\section{ADDENDUM}

The experimental technique described above has? been changed to prevent rupture at the graft $-\frac{T}{0}$ aortic suture line. More consistent long-term $\underset{\mathbb{\Phi}}{\circ}$ results are now obtained by placing the hetero- $\cong$ graft valve in a tube of Dacron and suturing the $\mathbb{\perp}$ tube in the descending aorta of the dog. 